\title{
Pacific
}

Journal of

Mathematics

\section{DESINGULARIZATIONS OF SOME UNSTABLE ORBIT} CLOSURES

\author{
MARK STEPHEN REEDER
}




\title{
DESINGULARIZATIONS OF SOME UNSTABLE ORBIT CLOSURES
}

\author{
MARK REEDER
}

Let $\sigma$ be a semisimple automorphism of a connected reductive group $G$, and let $G_{\sigma}$ be the fixed points of $\sigma$. We consider the $G_{\sigma}$-orbits on the space of nilpotent elements in an eigenspace of $d \sigma$. We give a desingularization of the orbit closures and relate the $G_{\sigma}$-orbits to the $G$-orbits. Along the way, we describe the fixed points of $\sigma$ on a flag variety $G / P$ where $P$ is a $\sigma$-stable parabolic subgroup of G.

I. Introduction. In this note we observe some consequences of Richardson's theorems on orbits of reductive groups, in the following situation. Let $G$ be a simply-connected reductive algebraic group over an algebraically closed field $F$ whose characteristic is either zero or sufficiently large (as specified below). Let $\mathfrak{g}$ be the Lie algebra of $G$, and let $\mathcal{N}$ be the variety of nilpotent elements in $\mathfrak{g}$. Let $\sigma$ be a semisimple automorphism of $G$, fix a nonzero element $q \in F^{\times}$, and consider the variety

$$
\mathcal{N}_{\sigma, q}=\{x \in \mathcal{N}: d \sigma(x)=q x\} .
$$

If $q$ is not a root of unity then $\mathcal{N}_{\sigma, q}$ is the whole $q$-eigenspace of $d \sigma$, hence is a linear subspace of $\mathfrak{g}$. If $q$ is a root of unity, the variety $\mathcal{N}_{\sigma, q}$ may even be reducible.

It was shown by Steinberg that the group of $\sigma$-fixed points $G_{\sigma}$ is also a connected reductive $F$-group $([\mathbf{S}])$. The adjoint action of $G_{\sigma}$ preserves each eigenspace of $d \sigma$, and $\mathcal{N}_{\sigma, q}$ consists of those $G_{\sigma^{-}}$ orbits in the $q$-eigenspace of $d \sigma$ which are "unstable", in the sense of geometric invariant theory $([\mathbf{H 2}])$. According to a theorem of Kac and Richardson ([Ri3]), the $G_{\sigma}$-orbits on $\mathcal{N}_{\sigma, q}$ are exactly the irreducible components of sets of the form $\mathcal{N}_{\sigma, q} \cap \tilde{\mathcal{O}}$, where $\tilde{\mathcal{O}}$ is a nilpotent $G$ orbit. Richardson also proved (with our assumptions on the characteristic of $F$, see [Ri1]) that there are only finitely many 
nilpotent $G$-orbits, hence there are only finitely many $G_{\sigma}$-orbits in $\mathcal{N}_{\sigma, q}$.

Let $\mathcal{O} \subseteq \mathcal{N}_{\sigma, q}$ be one such $G_{\sigma}$-orbit. Then the Zariski closure $\overline{\mathcal{O}}$ of $\mathcal{O}$ in $\mathcal{N}_{\sigma, q}$ is an affine variety which is generally singular. The main purpose of this note is to resolve the singularities of $\overline{\mathcal{O}}$ (Prop. (3.2)). More precisely, we construct a vector bundle $E$ over a partial flag variety of $G_{\sigma}$, and define a closed morphism $\pi: E \longrightarrow \overline{\mathcal{O}}$ such that $\pi: \pi^{-1}(\mathcal{O}) \longrightarrow \mathcal{O}$ is an isomorphism. The idea is simply that $\left(\sigma, q^{-1}\right) \quad$ acts on the known resolution of the closure of $G \cdot \mathcal{O}$, the nilpotent $G$-orbit containing $\mathcal{O}$. The desired resolution of $\overline{\mathcal{O}}$ is then found by taking fixed points under $\left(\sigma, q^{-1}\right)$. Moreover these fixed points separate the various $G_{\sigma}$-orbits in $\mathcal{N}_{\sigma, q} \cap G \cdot \mathcal{O}$, as described in Prop. (4.1) below. For example if $\sigma$ is induced by an automorphism of the Dynkin diagram, (4.1) implies that every nilpotent $G$-orbit meets $\mathcal{N}_{\sigma, q}$ in at most one $G_{\sigma}$-orbit. Taking $q=1$, we recover the well-known fact that nilpotent orbits in $\mathfrak{s o}(2 n+1)$ and $\mathfrak{s p}(n)$ are determined by elementary divisors.

The method requires a precise description (Prop. (2.3)) of the fixed points of $\sigma$ in a flag variety $G / P$ where $P$ is a $\sigma$-stable parabolic subgroup of $G$. Richardson ([Ri3] ) has already proven that there are finitely many orbits and they are all closed. Here we count and describe the orbits explicitly, using Steinberg's work in [S].

Hesselink ([H2]) has constructed desingularizations of closures of "strata" for more general group actions. Each stratum is a union of orbits, and in the case of nilpotent $G$-orbits, Kraft proved that the strata and orbits coincide. For our varieties $\mathcal{N}_{\sigma, q}$ it is not known if the orbits are strata, and even if they are (it is an interesting question), the proof is likely to be a more difficult route to a desingularization than the one taken here.

The group action $\left(G_{\sigma}, \mathcal{N}_{\sigma, q}, A d\right)$ arises in many settings. Suppose that $q$ is not a root of unity, so that $\mathcal{N}_{\sigma, q}$ is a linear space, and in particular an irreducible variety. There is a unique Zariski dense orbit in $\mathcal{N}_{\sigma, q}$, so by definition, the triple $\left(G_{\sigma}, \mathcal{N}_{\sigma, q}, A d\right)$ is a "prehomogeneous vector space", hereafter abbreviated as PV. The complex PV's which are also irreducible representations were classified by Sato and Kimura $([\mathbf{S}-\mathbf{K}])$. The PV's occuring as some $\left(G_{\sigma}, \mathcal{N}_{\sigma, q}, A d\right)$ are called "PV's of parabolic type" ([Ru]), because they also arise as subspaces of nilradicals of parabolic subalgebras which are in- 
variant under a Levi subgroup. ( $G_{\sigma}$ is a Levi subgroup in some reductive subgroup $H<G$ whose Lie algebra contains $\mathcal{N}_{\sigma, q}$.) Most irreducible PV's with finitely many orbits are of parabolic type. However, parabolic PV's can easily be reducible, and there seems to be no classification of reducible PV's. ${ }^{*}$ We remark that the big group $G$ is not to be discarded, as it greatly clarifies the structure of $\left(G_{\sigma}, \mathcal{N}_{\sigma, q}, A d\right)$.

Suppose now that $q \in F^{\times}$is arbitrary, but that $\sigma$ either has finite order or is conjugation by some element in a one-parameter subgroup of $G$. Then $\mathfrak{g}$ has a grading $\mathfrak{g}=\oplus \mathfrak{g}_{\imath}$ such that $G_{\sigma}$ has Lie algebra $\mathfrak{g}_{0}$ and $V$ is the set of nilpotent elements in $\mathfrak{g}_{1}$. These spaces were initially studied by Vinberg ([V]), who proposed a scheme for classifying the orbits.

My initial interest in such orbit closures $\overline{\mathcal{O}}$ came from the representation theory of $p$-adic groups $([\mathbf{K}-\mathbf{L}])$. In this setting, $G$ is a complex group (the "Langlands dual group"), $\sigma$ is inner, and $q$ is the cardinality of the residue field, hence not a root of unity. The intersection cohomology of $\overline{\mathcal{O}}$ is apparently related to multiplicities in unramified principal series representations of the $p$-adic group dual to $G$, just as with Schubert varieties and Verma modules (see $[\mathbf{G}],[\mathbf{R}],[\mathbf{Z}])$. In this context, Zelevinsky found desingularizations of $\mathcal{O}$ for $G=G L_{n}$ and used them to compute the intersection cohomology of some special $\overline{\mathcal{O}}$ 's, for which his resolution was "small" in the sense of $[\mathbf{G}-\mathbf{M}]$. The resolutions constructed in this paper are not always small.

An earlier version of this paper had $\sigma$ being inner, as above. However, in addition to other helpful comments, the referee pointed out Sekiguchi's paper [Se], which discusses $\mathcal{N}_{\sigma,-1}$ when $\sigma$ is an involution. Among other results, Sekiguchi gives a resolution of the orbit closures of maximal dimension in $\mathcal{N}_{\sigma,-1}$, so following the referee's suggestion, I modified this paper to include semisimple automorphisms, thus extending that part of Sekiguchi's work. In this setting, the $G_{\sigma}$-orbits in $\mathcal{N}_{\sigma,-1}$ are of interest in the representation theory of real Lie groups (see [Vo]).

Thanks are due to Gary Seitz, for telling me about Richardson's paper [Ri3].

*Added in proof: See Kasei-Kimura-Yasukura, Amer. J. Math., 108 (1986), 643-692. 
II. Fixed point varieties in flag manifolds. Here we use some of Steinberg's results in $[\mathbf{S}]$ to describe the fixed point subvariety of a flag manifold under the action of a semisimple automorphism. In this section there is no restriction on the characteristic of the ground field $F$.

Let $G$ be a connected, simply-connected and semisimple algebraic group over an algebraically closed field $F$, and let $\sigma$ be a semisimple automorphism of $G$. That is, $\sigma$ is an automorphism of $G$ whose differential $d \sigma$ acts diagonalizably on the Lie algebra $\mathfrak{g}$ of $G$. We say a subgroup $H \subseteq G$ is " $\sigma$-stable" if $\sigma H=H$. If $K \subseteq H \subseteq G$ are closed $\sigma$-stable subgroups of $G$, then $\sigma$ acts on the variety $H / K$ by the rule $\sigma(h K)=(\sigma h) K$, for $h \in H$. We write $(H / K)_{\sigma}$ for the fixed points of $\sigma$ in $H / K$. According to $[\mathbf{S},(8.2)], G_{\sigma}$ is a connected reductive group. By $[\mathrm{S},(7.5)]$, there exists a $\sigma$-stable Borel subgroup $B \subseteq G$, and a $\sigma$-stable maximal torus $T \subseteq B$. Let $U$ be the unipotent radical of $B$, and let $\mathfrak{t} \subseteq \mathfrak{t} \oplus \mathfrak{u}=\mathfrak{b}$ be the corresponding Lie algebras. Let $\Delta, \Delta^{+}, \Sigma$ be the roots of $\mathfrak{t}$ in $\mathfrak{g}$, $\mathfrak{u}$ and $\mathfrak{u} /[\mathfrak{u}, \mathfrak{u}]$, respectively. Since $\sigma$ preserves $T$ and $B$, it acts on $\Delta$, preserving $\Sigma$ and $\Delta^{+}$, and on the normalizer $N$ of $T$ in $G$, and hence on the Weyl group $W=N / T$. Let $W_{\sigma}^{1}=N_{\sigma} / T_{\sigma}$. This is the subgroup of $W_{\sigma}$ consisting of elements which can be represented in $N_{\sigma}$.

Let $V \subset \mathfrak{t}^{*}$ be the real span of the roots in $\Delta$, and let $V_{\sigma}$ be the fixed points of $\sigma$ in $V$. For any root $\alpha$, let $\bar{\alpha}$ denote its orthogonal projection into $V_{\sigma}$, with respect to a $W$-invariant inner product on $V$. Then $W_{\sigma}$ preserves and acts faithfully on $V_{\sigma}([\mathbf{S},(1.32)])$. Let $\overline{W_{\sigma}}$ and $\overline{W_{\sigma}^{1}}$ denote the restrictions of $W_{\sigma}$ and $W_{\sigma}^{1}$ to $V_{\sigma}$. We have $\overline{W_{\sigma}} \simeq W_{\sigma}$ as abstract groups, but the latter is a reflection group with respect to a new root system. We describe this more precisely.

The projections of all roots form a non-reduced root system in $V_{\sigma}$. We get a reduced root system as follows $([\mathbf{S}, \S 1])$. Let $S_{\alpha}$ be the collection of positive roots whose projection to $V_{\sigma}$ is proportional to $\bar{\alpha}$. There are two possibilities for $S_{\alpha}([\mathbf{S},(8.2)])$ :

(1) $S_{\alpha}=\left\{\alpha, \sigma \alpha, \sigma^{2} \alpha, \ldots\right\}$ no two of which sum to a root, or

(2) $S_{\alpha}=\{\alpha, \sigma \alpha, \beta=\alpha+\sigma \alpha\}$ is a $\sigma$-stable positive system of type $A_{2}$.

For $\alpha \in \Delta^{+}$, let $[\alpha] \in V_{\sigma}$ be the longest projection to $V_{\sigma}$ of a root in $S_{\alpha}$. Likewise, for any subset $J \subseteq \Delta^{+}$, let $[J]=\{[\alpha]: \alpha \in J\}$. 
Set $[\Delta]:=W_{\sigma}[\Sigma]$. Then by $[\mathbf{S},(1.32)],[\Delta]$ is a root system in $V_{\sigma}$ with base $[\Sigma]$, positive roots $[\Delta]^{+}=\left[\Delta^{+}\right]$and Weyl group $\overline{W_{\sigma}}$. (The root system of $G_{\sigma}$ turns out to be a subsystem of $[\Delta]$.) According to $[\mathbf{S},(8.2)]$, the reflection $s_{[\alpha]} \in \overline{W_{\sigma}}$ corresponding to $[\alpha] \in\left[\Delta^{+}\right]$is given by

(1) $s_{[\alpha]}=\left.s_{\alpha} s_{\sigma \alpha} \cdots\right|_{V_{\sigma}}$ or

(2) $s_{[\alpha]}=\left.s_{\beta}\right|_{V_{\sigma}}$,

according to the two cases for $S_{\alpha}$ described above.

All of this is related to the structure of fixed point group $G_{\sigma}$ in the following way. For each positive root $\alpha$, let $U_{\alpha} \subseteq U$ be the corresponding root subgroup. We have $\sigma U_{\alpha}=U_{\sigma \alpha}$. Consider the product $\prod_{\beta \in S_{\alpha}} U_{\beta}$. In the two cases for $S_{\alpha}$, either the root groups in the product commute (case (1)), or the whole product is the three dimensional Heisenberg group (case (2)). Hence the product is a group. Since $\sigma S_{\alpha}=S_{\alpha}$, the product is also invariant under $\sigma$, and we set

$$
U[\alpha]=\left(\prod_{\beta \in S_{\alpha}} U_{\beta}\right)_{\sigma} .
$$

By $[\mathbf{S},(8.2)]$ again, $U[\alpha]$ is either trivial, or a one-parameter group. Finally, $[\mathbf{S},(8.2)(4)-(7)]$ combine to give

LEMMA 2.1. $U[\alpha]$ is nontrivial if and only if the reflection $s_{[\alpha]}$ belongs to $\overline{W_{\sigma}^{1}}$. Moreover, $\overline{W_{\sigma}^{1}}$ is generated by such reflections.

This allows us to prove

LEMMA 2.2. There exists a set $Y(\sigma)$ of coset representatives for $W_{\sigma}^{1} \backslash W_{\sigma}$ such that if $n_{w} \in N$ represents $w \in Y(\sigma)$, then

$$
n_{w}^{-1} B_{\sigma} n_{w} \subseteq B
$$

Proof. Since $U_{\sigma}$ is the product of the $U[\alpha]$ 's, this amounts to having $n_{w}^{-1} U[\alpha] n_{w} \subseteq U$ for all $\alpha \in \Delta^{+}$. Since

$$
n_{w}^{-1} U[\alpha] n_{w} \subseteq \prod_{\beta \in S_{\alpha}} U_{w^{-1} \beta}
$$

it is enough to find coset representatives $w \in W_{\sigma}$ such that $w^{-1} S_{\alpha} \subseteq$ $\Delta^{+}$whenever $U[\alpha]$ is nontrivial. Let $<$ be the Bruhat order on the 
Weyl group $\overline{W_{\sigma}}$, with respect to the positive system $\left[\Delta^{+}\right]$of $[\Delta]$. This satisfies the rule $([\mathbf{J},(2.19)])$

$$
s_{[\alpha]} \bar{w}>\bar{w} \Leftrightarrow \bar{w}^{-1}[\alpha] \in\left[\Delta^{+}\right] .
$$

In each coset in $\overline{W_{\sigma}^{1}} \backslash \overline{W_{\sigma}}$, choose one element $\bar{w} \in \overline{W_{\sigma}}$ which is not $<$ any other member of its coset $\overline{W_{\sigma}^{1}} \bar{w}$. Let $w \in W_{\sigma}$ be the unique element whose restriction to $V_{\sigma}$ is $\bar{w}$, and let $Y(\sigma)$ be the collection of $w$ 's so obtained. Then $Y(\sigma)$ is a set of coset representatives for $W_{\sigma}^{1} \backslash W_{\sigma}$.

For any $x \in W_{\sigma}$, we have $x \sigma=\sigma x$, as automorphisms of $\Delta$. Let $\alpha \in \Delta^{+}$. Since $\sigma$ preserves $\Delta^{+}$and $S_{\alpha}$ is spanned over the positive integers by the $\sigma$-translates of one of its members, we have either $x S_{\alpha} \subseteq \Delta^{+}$or $x S_{\alpha} \subseteq-\Delta^{+}$. Moreover, the former possibility holds if and only if $\bar{x}[\alpha] \in[\Delta]^{+}$. Now take $x=w^{-1}$, where $w \in Y(\sigma)$. By (2.1) and the Bruhat-minimality of $w$, we have $x[\alpha] \in[\Delta]^{+}$whenever $U[\alpha]$ is nontrivial, so $w$ has the required properties.

Now let $P$ be a $\sigma$-stable parabolic subgroup of $G$. By [S, (7.5)], there is a maximal torus $T$ and a Borel subgroup $B$, both $\sigma$-stable, such that $T \subseteq B \subseteq P$. With notation and results as above, $P$ corresponds to a $\sigma$-stable subset of $\Sigma_{P} \subseteq \Sigma$. More precisely, $\Sigma_{P}$ consists of those simple roots which are roots of $t$ in the Lie algebra of the unique Levi subgroup $L$ of $P$ containing $T$. Since $\sigma T=T$ and $\sigma P=P$, we also have $\sigma L=L$, so $\Sigma_{P}$ is indeed $\sigma$-stable. Let $W_{P}$ be the subgroup of $W$ generated by the reflections in $\Sigma_{P}$. Then $W_{P}$ is also preserved by $\sigma$. The following result may be viewed as the determination of all $\sigma$-stable parabolic subgroups in the $G$ conjugacy class of $P$.

Proposition 2.3. Let $G$ be a semisimple simply connected algebraic group. Let $\sigma$ be a semisimple automorphism of $G$, and let $P$ be a $\sigma$-stable parabolic subgroup of $G$. Then with notation as above, (1) $(G / P)_{\sigma}=\amalg \quad G_{\sigma} w P / P$ (disjoint union). In par$w \in W_{\sigma}^{1} \backslash W_{\sigma} /\left(W_{P}\right)_{\sigma}$

ticular, each connected component of $(G / P)_{\sigma}$ is a $G_{\sigma}$-orbit.

(2) Each double coset $W_{\sigma}^{1} w\left(W_{P}\right)_{\sigma}$ meets $Y(\sigma)$ (see $(2.2)$ ).

(3) Each $G_{\sigma}$-orbit in $(G / P)_{\sigma}$ is complete. In other words, the group of $\sigma$-fixed points in any $\sigma$-stable parabolic subgroup of $G$ is parabolic in $G_{\sigma}$. 
(4) If $P=B$ is a $\sigma$-stable Borel subgroup and $y \in Y(\sigma)$, then $\left(y B y^{-1}\right)_{\sigma}=B_{\sigma}$, so $(G / B)_{\sigma}$ is a disjoint union of $\left[W_{\sigma}: W_{\sigma}^{1}\right]$ copies of the flag variety $G_{\sigma} / B_{\sigma}$.

Proof. Assertion (2) follows immediately from (2.2). For (1) we recall the Bruhat decomposition. For each $w \in W$, let $U_{w}$ be the product, taken in some fixed order, of the root groups $U_{\alpha}$ with $w^{-1} \alpha \in-\Delta^{+}$. Let $W^{P}$ be the set of $w \in W$ such that $w \Sigma_{P} \subseteq \Delta^{+}$. Then every point in $G / P$ may be uniquely written as $u w P$ for some $w \in W^{P}, u \in U_{w}$. Since $\Sigma_{P}$ and $\Delta^{+}$are $\sigma$-stable, so are $W^{P}$ and $U_{w}$. It follows that

$$
(G / P)_{\sigma}=\bigcup_{w \in W^{P}}\left(U_{w} w P / P\right)_{\sigma} \subseteq \bigcup_{w \in W_{\sigma}} U_{\sigma} w P / P \subseteq \bigcup_{w \in W_{\sigma}} G_{\sigma} w P / P .
$$

Let $n \in N$ represent some $w \in W_{\sigma}$. Then $\sigma(n)=n t$ for some $t \in T$, so $\sigma(w P)=\sigma(n) P=n t P=n P=w P$. Hence $w \in W_{\sigma}$ implies $G_{\sigma} w P / P \subseteq(G / P)_{\sigma}$, so we have

$$
(G / P)_{\sigma}=\bigcup_{w \in W_{\sigma}} G_{\sigma} w P / P=\bigcup_{y \in Y(\sigma)} G_{\sigma} y P / P,
$$

where $Y(\sigma)$ is as in (2.2).

We next show disjointness. Since $G=U N B=\amalg_{w \in W} U w B$, we have

$$
G_{\sigma}=U_{\sigma} N_{\sigma} B_{\sigma}=\coprod_{x \in W_{\sigma}^{1}} U_{\sigma} x B_{\sigma} .
$$

Let $y \in Y(\sigma)$ and consider the orbit $G_{\sigma} y P / P$ in $(G / P)_{\sigma}$. By (2.2) we have

$$
G_{\sigma} y P / P=\bigcup_{x \in W_{\sigma}^{1}} U_{\sigma} x B_{\sigma} y P / P=\bigcup_{x \in W_{\sigma}^{1}} U_{\sigma} x y P / P .
$$

It follows that for two elements $y, y^{\prime} \in Y(\sigma)$ we have $G_{\sigma} y P / P=$ $G_{\sigma} y^{\prime} P / P$ if and only if we have equality of double cosets

$$
W_{\sigma}^{1} y W_{P}=W_{\sigma}^{1} y^{\prime} W_{P}
$$

Since $Y(\sigma) \subseteq W_{\sigma}$, this is the same as

$$
W_{\sigma}^{1} y\left(W_{P}\right)_{\sigma}=W_{\sigma}^{1} y^{\prime}\left(W_{P}\right)_{\sigma},
$$


proving (1).

Now $(G / P)_{\sigma}$, being closed in $G / P$, is complete. Hence each connected component of $(G / P)_{\sigma}$ is complete, proving (3).

Finally, let $y \in Y(\sigma)$. Then $y^{-1} B_{\sigma} y \subseteq B$, so $B_{\sigma} \subseteq\left(y B y^{-1}\right)_{\sigma}$. Both sides are solvable, and are parabolic subgroups of $G_{\sigma}$ by (3). Hence they are Borel subgroups of $G_{\sigma}$, and must be equal.

2.5. Remarks. (1) Simple examples (eg. [Ri3, 3.3]) show that (2.3)(4) fails for nonminimal parabolic subgroups. See also (2.6) below.

(2) We could have given a more conceptual, though less explicit, construction of $Y(\sigma)$ by invoking [Ri3, 10.2.1] (our (2.3)(3)) at the outset. For, if $w \in W_{\sigma}$ and $B$ is a $\sigma$-stable Borel subgroup of $G$, then by $[\mathbf{R i 3}, 10.2 .1], B_{\sigma}$ and $\left(w B w^{-1}\right)_{\sigma}$ are Borel subgroups of $G_{\sigma}$ containing the same maximal torus $T_{\sigma}$, so there exists $x \in$ $W_{\sigma}^{1}$ such that $x^{-1}\left(B_{\sigma}\right) x=\left(w B w^{-1}\right)_{\sigma}$, from which it follows that $(x w)^{-1} B_{\sigma}(x w) \subseteq B$.

(3) Matsuki (over $\mathbb{C},[\mathbf{M}])$ and Springer $(\operatorname{char}(F) \neq 2$, $[\mathbf{S p}])$ have described all of the $G_{\sigma}$-orbits in $G / B$ when $\sigma$ is an involution. They prove in particular that $(G / B)_{\sigma}$ is exactly the union of the closed $G_{\sigma}$-orbits in $G / B$. Let $P$ be a parabolic subgroup containing $B$, and let $\pi: G / B \longrightarrow G / P$ be the natural projection. If $Y$ is a closed $G_{\sigma^{-}}$ orbit in $G / P$, then $Y=\pi\left(Y^{\prime}\right)$ for any closed $G_{\sigma}$-orbit $Y^{\prime} \subseteq \pi^{-1}(Y)$. Hence the result of Matsuki and Springer implies that $(G / P)_{\sigma}$ is the union of the closed $G_{\sigma}$-orbits. The same assertion holds when $\sigma$ is inner but not necessarily involutive. Indeed, $\sigma$ is then conjugation by some element of a torus $S \subseteq G_{\sigma}$. Let $Y \subseteq G / P$ be a closed $G_{\sigma}$-orbit as above. By Borel's fixed-point theorem [B, (10.4)], the fixed point set $Y_{S}$ of $S$ in $Y$ is nonempty, so $Y$ meets $(G / P)_{\sigma}$, hence $Y \subseteq(G / P)_{\sigma}$.

2.6. ExAmples. There are two extreme cases of (2.3). If $\sigma$ is conjugation by an element $s \in T$, then $W_{\sigma}=W$ and $W_{\sigma}^{1}$ is generated by the reflections about roots which are trivial on $s$. On the other hand if $\sigma$ is induced by an automorphism $\rho$ of the Dynkin diagram, then $W_{\sigma}^{1}=W_{\sigma}([\mathbf{S},(8.2)(5)])$, so $(G / P)_{\sigma}$ is connected.

Here is an example of a mixed case. Take $G=E_{6}$ (simply connected version), and let $\sigma$ be the involution of $G$ whose fixed point set has type $C_{4}$. Explicitly, take $\rho$ to be the automorphism of $E_{6}$ 
induced by the nontrivial diagram symmetry as in the previous paragraph, and let $s=\check{\alpha}_{0}(-1)$, where $\alpha_{0}$ is the highest root and $\check{\alpha}_{0}(t)$ is the corresponding one parameter subgroup of $T$. We can take $\sigma=i_{s} \rho$, where $i_{s}$ is conjugation by $s$. Then $W_{\sigma}$, which only depends on $\rho$, has type $F_{4}$. Meanwhile $W_{\sigma}^{1}$, being the Weyl group of $G_{\sigma}$, has type $C_{4}$. Hence $\left[W_{\sigma}: W_{\sigma}^{1}\right]=3$. We compute $Y(\sigma)$. Number the simple roots of $E_{6}$ as follows:

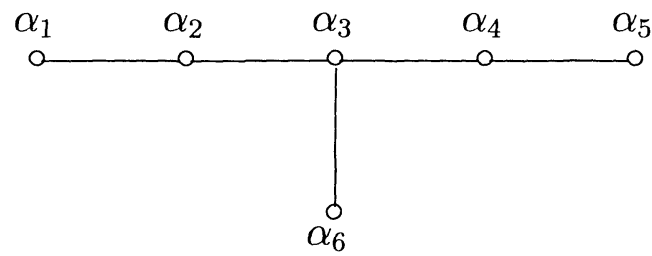

Using [S, (8.2)], one gets explicit conditions for each $U[\alpha]$ to be nontrivial, and the simple roots of $T_{\sigma}$ in $B_{\sigma}$ are then found to be

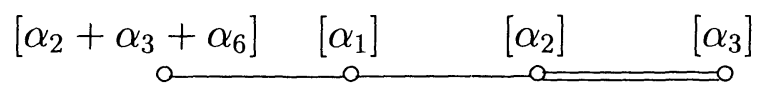

If $w \in Y(\sigma)$, we must have $w^{-1} \alpha>0$ for $\alpha=\alpha_{1}, \alpha_{2}, \alpha_{3}, \alpha_{2}+\alpha_{3}+\alpha_{6}$. (See the proof of (2.2).) Since $w$ is $\sigma$-invariant, we must also have $w^{-1} \alpha_{4}>0$ and $w^{-1} \alpha_{5}>0$. It is now easy to see that $w$ can only be one of $\left\{1, s_{6}, s_{6} s_{1}\right\}$, where $s_{i} \in W$ is the simple reflection about $\alpha_{i}$. Hence this set must be $Y(\sigma)$. Using $(2.3)$, we find that $(G / P)_{\sigma}$ has $3-\left|\Sigma_{P} \cap\left\{\alpha_{1}, \alpha_{6}\right\}\right|$ connected components. The components are not isomorphic in general. For example, let $P$ be a $\sigma$-stable parabolic subgroup with Levi component of type $A_{5}$. Then $P_{\sigma}$ has Levi of type $C_{3}$, while $\left(s_{6} P s_{6}^{-1}\right)_{\sigma}$ has Levi of type $A_{3}$, so $(G / P)_{\sigma}$ has two components: $G_{\sigma} / P_{\sigma} \simeq \mathbb{P}^{7}$ and $G_{\sigma} /\left(s_{6} P s_{6}^{-1}\right)_{\sigma}$, which is the variety of Lagrangian 4-planes in $F^{8}$.

III. The desingularization. We retain the notation and hypotheses of the previous section, except we now assume that the characteristic of the ground field $F$ is either zero or $p>2 \kappa$, where $\kappa$ is the maximum of the Coxeter numbers of the simple factors of $G$. For any variety $S$ on which $\sigma$ or $d \sigma$ acts, $S_{\sigma}$ denotes the fixed points. If $H$ is any $\sigma$-stable subgroup of $G$ with Lie algebra $\mathfrak{h}$, it 
follows from [B, III.9.1] that the Lie algebra of $H_{\sigma}$ is $\mathfrak{h}_{\sigma}$. We abbreviate the adjoint action of $G$ by $g \cdot Y:=A d(g) Y$ for $g \in G$ and any subset $Y \subseteq \mathfrak{g}$. Let $\mathcal{N}$ be the variety of nilpotent elements in the Lie algebra $\mathfrak{g}$ of $G$. Fix $q \in F^{\times}$, and set

$$
\mathcal{N}_{\sigma, q}=\{x \in \mathcal{N}: d \sigma(x)=q x\}
$$

We need the analogue of a normal $\mathfrak{s l}_{2}$-triple ([K-R, (I.2)]).

Lemma 3.1. Let $e \in \mathcal{N}_{\sigma, q}$. Then there exists $h \in \mathfrak{g}_{\sigma}, f \in \mathfrak{g}$ such that

$$
[h, e]=2 e, \quad[h, f]=-2 f, \quad[e, f]=h .
$$

Proof. The proof is a straightforward modification of ([K-R, Prop. 4]). By the version of the Jacobson-Morozov theorem given in [Ca, p. 152], there exists a triple $\left(e, h^{\prime}, f^{\prime}\right)$ satisfying the above relations. Write $h^{\prime}=\sum h_{\lambda}^{\prime}$ where $d \sigma\left(h_{\lambda}^{\prime}\right)=\lambda h_{\lambda}^{\prime}$, $\lambda \in F^{\times}$. Then $2 e=\left[h^{\prime}, e\right]=\sum\left[h_{\lambda}^{\prime}, e\right]$. Comparing $d \sigma$ eigenvalues, we get $2 e=\left[h_{1}^{\prime}, e\right]$. Likewise, we write $f^{\prime}=\sum f_{\lambda}^{\prime}$, and the relation $\left[e, f^{\prime}\right]=h^{\prime}$ implies $h_{1}^{\prime}=\left[e, f_{q^{-1}}^{\prime}\right] \in[e, \mathfrak{g}]$. Set $h=h_{1}^{\prime}$. By [Ko, Cor. 3.5] (which is shown in [Ca, p. 141] to apply when $\operatorname{char}(F)>2 \kappa)$, there exists $f$ such that $(e, h, f)$ satisfy the above relations.

Let $e \in \mathcal{N}_{\sigma, q}$ and fix a triple $(e, h, f)$ as in (3.1). We have a grading $\mathfrak{g}=\oplus_{i} \mathfrak{g}(i)$, where $\mathfrak{g}(i)=\{x \in \mathfrak{g}:[h x]=i x\}$. Each $\mathfrak{g}(i)$ is also $d \sigma$-stable, since $\operatorname{ad}(h)$ and $d \sigma$ commute. Hence the parabolic subalgebra

$$
\mathfrak{p}=\bigoplus_{i \geq 0} \mathfrak{g}(i)
$$

is $d \sigma$-stable, so the corresponding parabolic subgroup $P$ of $G$ is $\sigma$ stable, and we are in the situation of (2.3). In particular, $P_{\sigma}$ is a parabolic subgroup of the connected reductive group $G_{\sigma}$, and the Lie algebra of $P_{\sigma}$ is

$$
\mathfrak{p}_{\sigma}=\bigoplus_{i \geq 0} \mathfrak{g}(i)_{\sigma}
$$

We next set

$$
\mathfrak{p}^{2}=\bigoplus_{i \geq 2} \mathfrak{g}(i), \quad \mathfrak{q}=\mathcal{N}_{\sigma, q} \cap \mathfrak{p}^{2}
$$


The latter is an $\operatorname{Ad}\left(P_{\sigma}\right)$-invariant linear subspace of $\mathcal{N}_{\sigma, q}$, even if $q$ is a root of unity, because $\mathfrak{p}^{2}$ consists of nilpotent elements. Let

$$
\mathfrak{p}_{0}^{2} \subset \mathfrak{p}^{2}
$$

be the $P$-orbit of $e$. By [Ca, 5.7.3], $\mathfrak{p}_{0}^{2}$ is the unique open dense $P$-orbit in $\mathfrak{p}^{2}$. It follows that $\mathfrak{p}_{0}^{2}$ and hence $G \cdot e$ are $d \sigma$ stable.

Define the incidence variety

$$
E=\left\{\left(g P_{\sigma}, x\right) \in G_{\sigma} / P_{\sigma} \times \mathfrak{g}: x \in g \cdot \mathfrak{q}\right\} .
$$

This is a vector bundle over $G_{\sigma} / P_{\sigma}$ via projection onto the first factor. One could view $E$ as the fiber product $G_{\sigma} \times_{P_{\sigma}} \mathfrak{q}$, but the former picture is more convenient here. Let

$$
\pi: E \longrightarrow \mathcal{N}_{\sigma, q}
$$

be projection onto the second factor. Then $\pi$ is $G_{\sigma}$-equivariant, and closed since $G_{\sigma} / P_{\sigma}$ is complete, by $(2.3)(3)$.

Proposition 3.2. Let $\mathcal{O}$ be the $G_{\sigma}$-orbit of $e \in \mathcal{N}_{\sigma, q}$. Then the image of $\pi$ is $\overline{\mathcal{O}}$ and $E \stackrel{\pi}{\rightarrow} \overline{\mathcal{O}}$ is a resolution of singularities.

Proof. We first show that the $P_{\sigma}$-orbit of $e$ is dense in $\mathfrak{q}$. From the representation theory of $S L_{2}([\mathbf{C a}, 5.4])$ we see that the map

$$
\operatorname{ad}(e): \mathfrak{g}(i) \longrightarrow \mathfrak{g}(i+2)
$$

is surjective for $i \geq 0$. Hence, given $x \in \mathfrak{q}$, there exists $y \in \mathfrak{p}$ with $[y, e]=x$. We can write $y=\Sigma y^{\lambda}$ where $y^{\lambda} \in \mathfrak{p}$ and $d \sigma\left(y^{\lambda}\right)=\lambda y^{\lambda}$ for $\lambda \in F^{\times}$. Comparing eigenvalues of $d \sigma$, we get $x=\left[y^{1}, e\right]$, and $y^{1} \in \mathfrak{p}_{\sigma}$. This shows that $\operatorname{ad}(e): \mathfrak{p}_{\sigma} \longrightarrow \mathfrak{q}$ is surjective, so $P_{\sigma} \cdot e$ is open and dense in $\mathfrak{q}$.

From this it follows easily that the image of $\pi$ is $\overline{\mathcal{O}}$. Indeed, since the image of $\pi$ is closed and contains $e$, we have $\overline{\mathcal{O}} \subseteq \pi(E)$. On the other hand,

$$
\mathfrak{q}=\overline{P_{\sigma} \cdot e} \subseteq \overline{\mathcal{O}} .
$$

Since $\pi(E)=G_{\sigma} \cdot \mathfrak{q}$ and $\overline{\mathcal{O}}$ is preserved by $G_{\sigma}$, we have the other containment.

We next show that $\pi$ is bijective over $\mathcal{O}$. We recall the well-known resolution of $\overline{G \cdot e}$ (c.f. [H, p. 108]). Consider the vector bundle

$$
\tilde{E}=\left\{(g P, x) \in G / P \times \mathfrak{g}: x \in g \cdot \mathfrak{p}^{2}\right\} .
$$


Then the map

$$
\tilde{E} \stackrel{\tilde{\pi}}{\rightarrow} \mathfrak{g}
$$

given by projection onto the second factor is $G$-equivariant, closed, has image $\overline{G \cdot e}$, and is bijective over $G \cdot e$.

We define an automorphism $\sigma_{q}$ of $\tilde{E}$ by

$$
\sigma_{q}(g P, x)=\left(\sigma(g) P, q^{-1} d \sigma(x)\right) .
$$

The fixed point space of $\sigma_{q}$ is

$$
\tilde{E}_{\sigma_{q}}=\left\{(g P, x) \in(G / P)_{\sigma} \times \mathfrak{g}: x \in \mathcal{N}_{\sigma, q} \cap g \cdot \mathfrak{p}^{2}\right\} .
$$

This is a disjoint union of vector bundles over the connected components of $(G / P)_{\sigma}$. In the notation of (2.3) these components are given by

$$
(G / P)_{\sigma}=\coprod_{w \in W_{\sigma}^{1} \backslash W_{\sigma} /\left(W_{P}\right)_{\sigma}} G_{\sigma} w P / P,
$$

and each component is a flag variety for $G_{\sigma}$.

The component of $\tilde{E}_{\sigma_{q}}$ lying over $G_{\sigma} P / P$ is

$$
\left\{(g P, x) \in G_{\sigma} P / P \times \mathfrak{g}: x \in \mathcal{N}_{\sigma, q} \cap g \cdot \mathfrak{p}^{2}\right\} .
$$

Since $G_{\sigma} / P_{\sigma}=G_{\sigma} P / P$ and for $g \in G_{\sigma}$, we have $\mathcal{N}_{\sigma, q} \cap g \cdot \mathfrak{p}^{2}=$ $g \cdot\left(\mathcal{N}_{\sigma, q} \cap \mathfrak{p}^{2}\right)=g \cdot \mathfrak{q}$, this component is none other than $E$.

Note that $\tilde{\pi}$ restricted to $E$ is just our original $\pi: E \longrightarrow \overline{\mathcal{O}}$. Since the fibers of $\tilde{\pi}$ over $G \cdot e$ are singletons, the same must be true of the fibers of $\pi$ over $\mathcal{O}$. The proof of (3.2) is now complete if the characteristic of $F$ is zero.

In sufficiently large characteristic, we use an argument from [Ri1] to prove that $\pi$ is separable. The assumption that $F$ has characteristic $p>2 \kappa$ is, by a wide margin, enough to ensure (see [Ri1,$\S 5]$ ) that the orbit maps under the adjoint action of $G$ are separable. Hence the tangent space to $G \cdot e$ is $T_{e} G \cdot e=[\mathfrak{g}, e]$. Decompose $\mathfrak{g}=\oplus \mathfrak{g}^{\lambda}$ into $d \sigma$-eigenspaces, so $\mathcal{O} \subseteq \mathfrak{g}^{q}$. Then $T_{e} \mathcal{O} \subseteq \mathfrak{g}^{q} \cap T_{e} G e=\mathfrak{g}^{q} \cap \oplus_{\lambda}\left[\mathfrak{g}^{\lambda}, e\right]=$ $\left[\mathfrak{g}_{\sigma}, e\right]$. It follows that the differential of $\pi$ at $\left(P_{\sigma}, e\right) \in E$ is surjective, so $\pi$ is separable by [B, p. 41]. Hence $\pi: \pi^{-1}(\mathcal{O}) \longrightarrow \mathcal{O}$ is an isomorphism by Zariski's Main Theorem.

Corollary 3.3 .

(1) $\operatorname{dim} \mathcal{O}=\operatorname{dim} G_{\sigma}-\operatorname{dim} P_{\sigma}+\operatorname{dim} \mathfrak{q}$. 
(2) $C_{G_{\sigma}}(e)=C_{P_{\sigma}}(e)$, where $C_{H}(e)$ denotes centralizer in $H$ of $e$.

(3) $\mathcal{O} \cap \mathfrak{q}$ is exactly the dense $P_{\sigma}$-orbit on $\mathfrak{q}$. (See the proof of (3.2).)

Proof. The first two assertions are immediate from (3.2). We use them to compute

$$
\begin{aligned}
\operatorname{dim} P_{\sigma} \cdot e & =\operatorname{dim} P_{\sigma}-\operatorname{dim} C_{P_{\sigma}}(e) \\
& =\operatorname{dim} P_{\sigma}-\operatorname{dim} C_{G_{\sigma}}(e) \\
& =\operatorname{dim} P_{\sigma}+\operatorname{dim} \mathcal{O}-\operatorname{dim} G_{\sigma} \\
& =\operatorname{dim} P_{\sigma}+\left[\operatorname{dim} G_{\sigma}+\operatorname{dim} \mathfrak{q}-\operatorname{dim} P_{\sigma}\right]-\operatorname{dim} G_{\sigma} \\
& =\operatorname{dim} \mathfrak{q} .
\end{aligned}
$$

It follows that $P_{\sigma} \cdot e$ contains an open subset of $\mathfrak{q}$ which must meet, hence equal, the dense $P_{\sigma}$ orbit on $\mathfrak{q}$.

According to $[\mathbf{K}]$, a result like (3.2) has the following consequence in characteristic zero. Let $\mathcal{O}$ be an $G_{\sigma}$-orbit in $\mathcal{N}_{\sigma, q}$, and let $E$ be the bundle over $G_{\sigma} / P_{\sigma}$ as in (3.2). Let $S^{d} \check{\mathcal{E}}$ be the graded sheaf of sections of the $d^{\text {th }}$ symmetric power of the dual bundle of $E$. Then $\oplus_{d \geq 0} S^{d} \check{\mathcal{E}}$ is the structure sheaf $\mathcal{O}_{E}$ of $E$, and the global sections $H^{0}\left(G_{\sigma} / P_{\sigma}, S^{d} \check{\mathcal{E}}\right)$ are the regular functions on $E$ which are polynomial of degree $d$ on each fiber. Let $F[\overline{\mathcal{O}}]$ be the coordinate ring of the affine variety $\overline{\mathcal{O}}$. The map $\pi$ induces an injection of $G_{\sigma}$-modules

$$
\pi^{*}: F[\overline{\mathcal{O}}] \hookrightarrow \bigoplus_{d \geq 0} H^{0}\left(G_{\sigma} / P_{\sigma}, S^{d} \check{\mathcal{E}}\right)
$$

given by $\pi^{*}(f)\left(m P_{\sigma}\right)=\left.f\right|_{m \cdot q}$.

Corollary 3.4. Assume the characteristic of $F$ is zero and that $\mathfrak{q}$ is a completely reducible $P_{\sigma}$-module. Then

(1) $\pi^{*}$ is an isomorphism of $G_{\sigma}$-modules.

(2) $\overline{\mathcal{O}}$ is a normal variety.

(3) $H^{i}\left(E, \mathcal{O}_{E}\right)=0$ for $i>0$. (One says that $\overline{\mathcal{O}}$ has "rational singularities").

The proof of the analogous result for nilpotent orbits in $[\mathbf{H}]$ carries over without change. Note that $\mathfrak{q}$ is a completely reducible $P_{\sigma^{-}}$ module if and only if the unipotent radical of $P_{\sigma}$ acts trivially on q. 
IV. From $G$-orbits to $G_{\sigma}$-orbits. We retain the notation from the proof of (3.2), and investigate the other components of $\tilde{E}_{\sigma_{q}}=$ $\left(G \times{ }_{P} \mathfrak{p}^{2}\right)_{\sigma_{q}}$ occurring there. For $w \in W_{\sigma}$, we put

$$
\mathfrak{q}_{w}=\mathcal{N}_{\sigma, q} \cap w \cdot \mathfrak{p}^{2}
$$

so $\mathfrak{q}=\mathfrak{q}_{1}$. Note that $\mathfrak{q}_{w}$ is an $\operatorname{Ad}\left(w P w^{-1}\right)_{\sigma}$-invariant linear subspace of $\mathcal{N}_{\sigma, q}$. It follows from (2.3) that for each $w \in W_{\sigma}^{1} \backslash W_{\sigma} /\left(W_{P}\right)_{\sigma}$ we have a component

$$
E_{w}=\left\{(g w P, x) \in G_{\sigma} w P / P \times \mathfrak{g}: x \in g \cdot \mathfrak{q}_{w}\right\},
$$

and

$$
\tilde{E}_{\sigma_{q}}=\coprod_{w \in W_{\sigma}^{1} \backslash W_{\sigma} /\left(W_{P}\right)_{\sigma}} E_{w} .
$$

One could also view $E_{w}$ as the fiber product

$$
E_{w}=G_{\sigma} \times{ }_{P_{\sigma}} \mathfrak{q}_{w}
$$

Since $E_{w}$ is a vector bundle over a smooth variety and $\tilde{\pi}$ is $G_{\sigma^{-}}$ equivariant and closed, we see that $\pi\left(E_{w}\right)=G_{\sigma} \cdot \mathfrak{q}_{w}$ is an $G_{\sigma}$-stable, closed, irreducible subvariety of $\mathcal{N}_{\sigma, q} \cap \overline{G \cdot e}$. Hence $\pi\left(E_{w}\right)=\overline{\mathcal{O}_{w}}$ for a unique $G_{\sigma}$-orbit $\mathcal{O}_{w} \subseteq G \cdot e$. Note that $\pi\left(E_{w}\right)$ meets $G \cdot e$ if and only if $\mathcal{N}_{\sigma, q} \cap w \cdot \mathfrak{p}_{0}^{2} \neq \varnothing$. (Recall that $\mathfrak{p}_{0}^{2}=\mathfrak{p}^{2} \cap G \cdot e$ is the unique dense $P$-orbit in $\mathfrak{p}^{2}$.) By $[\mathbf{R i 3}]$ the $G_{\sigma}$-orbits in $\mathcal{N}_{\sigma, q} \cap G \cdot e$ are exactly the irreducible components of $\mathcal{N}_{\sigma, q} \cap G \cdot e$. Thus, the closure of each such $G_{\sigma}$-orbit is an irreducible component of $\mathcal{N}_{\sigma, q} \cap \overline{G \cdot e}$. The main result in this section ties these considerations together and, in tandem with (2.3), reduces the computation of the $G_{\sigma}$-orbits in $G \cdot e$ to knowing the dense orbit in the prehomogeneous vector space $\left(P, \mathfrak{p}^{2}, \mathrm{Ad}\right)$. We note that for each $G$ there are only finitely many of the latter to consider.

Proposition 4.1. (1) The map $w \mapsto \mathcal{O}_{w}$ described above is a bijection from

$$
\left\{w \in W_{\sigma}^{1} \backslash W_{\sigma} /\left(W_{P}\right)_{\sigma}: \mathcal{N}_{\sigma, q} \cap w \cdot \mathfrak{p}_{0}^{2} \neq \varnothing\right\}
$$

to the set of $G_{\sigma}$-orbits in $\mathcal{N}_{\sigma, q} \cap G \cdot e$. The inverse of this bijection sends a $G_{\sigma}$-orbit $\mathcal{O} \subseteq G \cdot$ e to the $w \in W_{\sigma}^{1} \backslash W_{\sigma} /\left(W_{P}\right)_{\sigma}$ such that $E_{w}$ 
is the unique component of $\tilde{E}_{\sigma_{q}}$ meeting a $\tilde{\pi}$-fiber over some point in $\mathcal{O}$.

(2) The map $\pi: E_{w} \longrightarrow \overline{\mathcal{O}_{w}}$ is a desingularization of $\overline{\mathcal{O}_{w}}$.

(3) If $\mathcal{N}_{\sigma, q} \cap w \cdot \mathfrak{p}_{0}^{2}$ is nonempty, then $\mathcal{O}_{w}=G_{\sigma} \cdot\left(\mathcal{N}_{\sigma, q} \cap w \cdot \mathfrak{p}_{0}^{2}\right)=$ $\overline{\mathcal{O}_{w}} \cap G \cdot e$.

Proof. Since $\tilde{\pi}$ is bijective over $G \cdot e$, it is clear that any $G_{\sigma}$-orbit in $\mathcal{N}_{\sigma, q} \cap G \cdot e$ can meet $\pi\left(E_{w}\right)$ for at most one $w \in W_{\sigma}^{1} \backslash W_{\sigma} /\left(W_{P}\right)_{\sigma}$. Hence $w \mapsto \mathcal{O}_{w}$ is injective.

Take $x \in \mathcal{N}_{\sigma, q} \cap G \cdot e$, and look at the fiber $\tilde{\pi}^{-1}(x)=\{(g P, x)\}$, where $x \in g \cdot \mathfrak{p}^{2}$. We have $q x=d \sigma(x) \in d \sigma\left(g \cdot \mathfrak{p}^{2}\right)=\sigma(g) \cdot \mathfrak{p}^{2}$, so $x \in \sigma(g) \cdot \mathfrak{p}^{2}$. But then $(\sigma(g) P, x) \in \tilde{\pi}^{-1}(x)$, so $g P \in(G / P)_{\sigma}$. This shows that $\mathcal{N}_{\sigma, q} \cap G \cdot e$ is contained in the union of the $\pi\left(E_{w}\right)$ 's. Now consider a $G_{\sigma}$-orbit $\mathcal{O}_{0} \subseteq \mathcal{N}_{\sigma, q} \cap G \cdot e$, and suppose $\mathcal{O}_{0}$ meets $\pi\left(E_{w}\right)$. Then $\mathcal{O}_{0} \subseteq \pi\left(E_{w}\right)$ so $\overline{\mathcal{O}_{0}} \subseteq \pi\left(E_{w}\right)$ since the latter is closed. Recall however, that $\overline{\mathcal{O}_{0}}$ is an irreducible component of $\mathcal{N}_{\sigma, q} \cap \overline{G \cdot e}$, so we must have $\overline{\mathcal{O}_{0}}=\pi\left(E_{w}\right)$, since the latter is also irreducible.

Assertion (2) follows from the birationality of $\tilde{\pi}$, as we have argued before.

Let $x \in \overline{\mathcal{O}_{w}} \cap G e=G_{\sigma} \mathfrak{q}_{w} \cap G e$. Then there exist $m \in G_{\sigma}, g \in G$ and $v \in \mathfrak{p}^{2}$ such that $x=m w \cdot v=g \cdot e$, so $v=(m w)^{-1} g \cdot e \in \mathfrak{p}^{2} \cap G \cdot e=\mathfrak{p}_{0}^{2}$. Hence $x \in \mathcal{N}_{\sigma, q} \cap m w \cdot \mathfrak{p}_{0}^{2}$. This proves the second equality in (3), the other direction there being trivial.

We now know $\mathcal{O}_{w} \subseteq G_{\sigma} \cdot\left(\mathcal{N}_{\sigma, q} \cap w \cdot \mathfrak{p}_{0}^{2}\right)=\overline{\mathcal{O}_{w}} \cap G \cdot e$. Moreover, $\mathcal{N}_{\sigma, q} \cap w \cdot \mathfrak{p}_{0}^{2}$, being open in the linear space $\mathcal{N}_{\sigma, q} \cap w \cdot \mathfrak{p}^{2}$, is irreducible. It follows that $G_{\sigma} \cdot\left(\mathcal{N}_{\sigma, q} \cap w \cdot p_{0}^{2}\right)$ is an irreducible subset of $\mathcal{N}_{\sigma, q} \cap G \cdot e$ containing the irreducible component $\mathcal{O}_{w}$ of $\mathcal{N}_{\sigma, q} \cap G \cdot e$. Hence $\mathcal{O}_{w}=G_{\sigma} \cdot\left(\mathcal{N}_{\sigma, q} \cap w \cdot \mathfrak{p}_{0}^{2}\right)$.

Corollary 4.2. Let $\tilde{\mathcal{O}} \subset \mathfrak{g}$ be a nilpotent $G$-orbit. Then $\tilde{\mathcal{O}} \cap \mathcal{N}_{\sigma, q}$ is a union of at most $\left|W_{\sigma}^{1} \backslash W_{\sigma} /\left(W_{P}\right)_{\sigma}\right|$ orbits under $G_{\sigma}$.

Corollary 4.3. Assume $\sigma$ is induced by a symmetry of the Dynkin diagram of $G$. Let $\tilde{\mathcal{O}} \subset \mathfrak{g}$ be a nilpotent $G$-orbit. Then $\tilde{\mathcal{O}}$ meets $\mathcal{N}_{\sigma, q}$ in at most one $G_{\sigma}$-orbit.

Proof. In this situation, $W_{\sigma}^{1}=W_{\sigma}$ as remarked in (2.6), so the result is a special case of (4.3).

When $q=1$, we are counting the number of nilpotent $G_{\sigma}$-orbits in $\mathfrak{g}_{\sigma}$ which are contained in a single $G$-orbit. It is well-known that 
nilpotent orbits in $\mathfrak{s o}(2 n+1)$ and $\mathfrak{s p}(n)$ are determined by elementary divisors. The above results say this is due to the connectedness of the varieties $(G / P)_{\sigma}$, which is in turn deduced from the equality $W_{\sigma}^{1}=W_{\sigma}$. Likewise, every $\mathfrak{e}_{6}$ nilpotent orbit meets $\mathfrak{f}_{4}$ in at most one $F_{4}$-orbit, and similarly for $G_{2} \subset \operatorname{Spin}(8)$. In the case $G=S L(2 n), G_{\sigma}=S O(2 n)$, one checks that $|Y(\sigma)|=2$, so (4.2) reduces to the well-known result that at most two nilpotent orbits in $\mathfrak{s o}(2 n)$ have the same elementary divisors, and these orbits are conjugate in $O(2 n)$.

\section{REFERENCES}

[B] A. Borel, Linear Algebraic Groups, Springer-Verlag, 1991.

[Ca] R. Carter, Finite Groups of Lie Type: Conjugacy Classes and Complexc Characters, Wiley, 1985.

[G-M] M. Goresky and R. MacPherson, Intersection homology II, Invent. Math., 72 (1983), 77-129.

[G] V. Ginsburg, Proof of Deligne-Langlands conjecture, Doklady, 35 (1987), 304-308.

[H] W. Hesselink, The normality of closures of orbits in a Lie algebra, Comment. Math. Helv., 54 (1979), 105-110.

[H2] — Desingularizations of varieties of nullforms, Invent. Math., $\mathbf{5 5}$ (1979), 141-163.

[J] J. C. Janzten, Moduln mit Einem Höchsten Gewicht, vol. 750, SpringerVerlag.

[K-L] D. Kazhdan and G. Lusztig, Proof of the Deligne-Langlands conjecture for Hecke algebras, Invent. Math., 87 (1987), 153-215.

[K] G. Kempf, On the collapsing of homogeneous bundles, Invent. Math., 37 (1976), 229-239.

[Ko] B. Kostant, The principal three dimensional subgroup and the Betti numbers of a complex simple Lie group, Amer. J. Math., 81 (1959), 973-1032.

[K-R] B. Kostant and S. Rallis, Orbits and representations associated with symmetric spaces, Amer. J. Math., 93 (1971), 753-809.

[M] T. Matsuki, The orbits of affine symmetric spaces under the action of minimal parabolic subgroups, J. Math. Soc. Japan, 31 (1979), 331-357.

[R] M. Reeder, Whittaker functions, prehomogeneous vector spaces and standard representations of p-adic groups, J. reine angew. Math., (1994), 83-121.

[Ri1] R. W. Richardson, Conjugacy classes in Lie algebras and algebraic groups, Ann. Math., 86 (1967), 1-15.

[Ri2] _ Conjugacy classes in parabolic subgroups of semisimple algebraic 
groups, Bull. London Math. Soc., 6 (1974), 21-24.

[Ri3] - On orbits of algebraic groups and Lie groups, Bull. Austral. Math. Soc., 25 (1982), 1-28.

[Ru] H. Rubenthaler, Espaces vectoriels préhomogènes, sous-groupes paraboliques et sl $l_{2}$-triplets, C.R. Acad. Sci. Paris, Sér. A, 209 (1980), 127-129.

[S-K] M. Sato and T. Kimura, A classification of irreducible prehomogeneous vector spaces and their relative invariants, Nagoya Math. J., 65 (1977), $1-155$.

[Se] J. Sekiguchi, The nilpotent subvariety of the vector space associated to a symmetric pair, Publ. RIMS, Kyoto Univ., 20 (1984), 155-212.

[Sp] T. A. Springer, Some results on algebraic groups with involutions, Adv. Stud. Pure Math., vol. 6, Kinokuniya, North-Holland, 1985, 525-543.

[S] R. Steinberg, Endomorphisms of algebraic groups, Mem. Amer. Math. Soc., 80 (1968).

[V] E. B. Vinberg, On the classification of nilpotent elements in graded Lie algebras, Doklady, 16 (1975), 1517-1520.

[Vo] D. Vogan, Associated Varieties and Unipotent Representations, Representations of reductive groups, Birkhauser 1991.

[Z2] A.V. Zelevinsky, A p-adic analogue of the Kazhdan-Lusztig conjecture, Funktsional. Anal. i Prilozhen., 15 (1981), 9-21.

Received September 17, 1992 and in revised form January 12, 1993. Research partially supported by NSF grant DMS-9104183.

\section{UNIVERSITY OF OKLAHOMA}

NORMAN, OKLAHOMA 73019

E-mail address: mreeder@nsfuvax.math.uoknor.edu 




\section{PACIFIC JOURNAL OF MATHEMATICS}

Volume $167 \quad$ No. $2 \quad$ February 1995

Existence of shortest directed networks in $\mathbb{R}^{2}$

201

MANUEL AlFARO GARCIA

Hecke characters of singular Drinfel'd modules

215

SUNGHAN BAE

Factorization method for a bimeromorphic morphism

231

Jose PEREZ BLANCO

$L^{p}$ estimates for operators associated to flat curves without the Fourier 243 transform

Anthony Carbery, James Thomas Vance, JR., Stephen

WAINGER, DAVID K. WATSON and JAMES WRIGHT

$S$-integer points on elliptic curves

263

ROBERT HOWARD GROSS and JOSEPH SILVERMAN

On metrics defined by modules

JAMES ALLISTER JENKINS

Conditional Wiener integrals. II

ChUll PARK and DAVID LeE SKOUG

On a Plancherel formula for certain discrete, finitely generated, torsion-free nilpotent groups

CAROLYN PFEFFER JOHNSTON

Desingularizations of some unstable orbit closures

MARK STEPHEN REEDER

Determining multiplicities of half-integral weight newforms

Thomas Richard SHEMANSKE and LyNne WALLING

Generation of integral orthogonal groups over dyadic local fields 